Linköping Studies in Science and Technology Thesis No. 1712

\title{
Optical Studies of Materials for Spectral Design
}

\author{
Christina Åkerlind
}

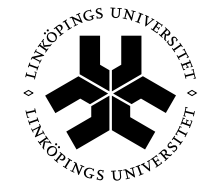

\section{Linköpings universitet \\ INSTITUTE OF TECHNOLOGY}

Department of Physics, Chemistry and Biology (IFM)

Linköping University, SE-581 83 Linköping, Sweden

Linköping 2015 
ISBN 978-91-7519-106-5

ISSN 0280-7971

Printed by LiU-Tryck, Linköping 2015 


\section{Abstract}

Optical material properties have been studied in a wide wavelength range. The aim is future use of spectral design for camouflage. The main characterization techniques used in this work are Reflection Spectroscopy, Scatterometry (BRDF) and Mueller Matrix Ellipsometry. Six camouflage evaluation criteria based on reflection, emissivity, polarization, gloss, dynamic coloring and broad band properties, are more or less related to the interaction between light and materia. Almost all are connected to the work of this thesis and are exemplified through different material categories with potential for use in camouflage applications. The included papers presents: a broad band (visible-infrared-microwave) study, two examples of dynamic optical properties of thin films, and polarization and scattering properties of a natural surface. 


\section{Preface}

This thesis is a part of my $\mathrm{PhD}$ studies at Laboratory of Applied Optics, Department of Physics, Chemistry and Biology (IFM), Linköping University, where I am enrolled as a part-time student within the work as a scientist at FOI (Swedish Defence Research Agency). As a PhD student I have been enrolled in the research schools Forum Securitatis and Agora Materiae at Linköpings University.

My topic is on camouflage related materials issues and some results from the years past are collected here. The theme is wide, the electromagnetic range covered is broad, and when you scratch on the surface it may seem shallow. But remember that, as in the rest of the world, it is almost only the surface that matters...

The word camouflage has been used since late 19-th century. It is thought to originate from the French word camoufler (to disguise) via a combination of the Italian camuffare and a French wording for the practical joke to "whiff smoke in the face"- camouflet.

And that is what it is about, to mock with colors to look alike, to confound with structures and reflections to not be looked at, but hide. In the ancient game hide and seek, for children a fun game, but all sincere war fighters know the ability to hide it is often the difference between life and death.

Today there is a challenging duel between the development of the camouflage materials (signature management technology) on the hiding side, and sensors of different kinds and combinations, threatening with detection, revealing and identification, on the seekers side. With emerging sensor technology, the hiding becomes trickier while the seekers always seem to be one step ahead. Despite the advances in technical development of sensors there are still limitations giving hope for camouflage management. However, to not be defeated, a vast improvement of camouflage materials and signature technology is needed.

Will the real "game" develop to an almost fictive reality where the urge towards invisibility as the ultimate camouflage, only seen its alike in tales and in the recent science fiction and fantasy movies expressed through examples as the invisible car of James Bond ${ }^{1}$ and the invisibility cloak of Harry Potter?

Future will tell!

\footnotetext{
${ }^{1}$ Die Another Day (2002), directed by Lee Tamahori.

${ }^{2}$ Harry Potter and the Philosopher's Stone is the first of the book of seven written by J.K. Rowling.
} 


\section{Acknowledgements}

This work has been a long and tortuous journey and the route was not obvious to start with. (Is it possible to make it look straight with hindsight?) As this type of research is not one-persons work, I would like to thank:

-My supervisors Professor Kenneth Järrendahl, Professor Hans Arwin and Doctor Hans Kariis for adopting me as a student, for patience and scientific challenges. It is a pleasure working with you!

-The Armed Forces for financial support.

-Co-writers Eva H Karlsson, Anna Jänis, Fredrik Jakobsson, Kent Andersson, Jan Landin, Johan Gustafsson, Tomas Hallberg. Without you the articles included would not have been the same and the work not as enjoyable.

-Colleges at IFM and Laboratory of Applied Optics to name a few: Torun Berlind, Roger Magnusson, Anna Maria Uhlin, Lía Fernández del Río, Iryna Valyukh, Sergiy Valyukh, for the friendly environment, encouragements and for making me feel so welcome when I now and then pop up like "jack in the box".

-Colleges at FOI in Linköping, especially the D1.1-corridor mates through the years, for the inspiring environment and great corridor-, and friendship, the frequent $\mathrm{RPS}^{3}$-players for good lunch-company, great fellowship, fair play and the coffee.

To name a few, without internal ranking: Anna Pohl, Annelie Längerberg, Erik Zdansky, Gunilla Friberg, Jan Fagerström, Ronny Gunnarsson, Sandra Hultman, Cesar Lopes, Lars Kroon, Lars Pettersson, Magnus Höijer, Nils Karlsson, Niklas Wellander, Patrick Andersson, Stefan Björkert, Stefan Persson, Steven Savage, Tomas Boman, Tommy Johansson, Wivianne Asp, Åsa Andersson.

-Thanks to family and friends for your encouragements, Andreas for your support and for sharing life with me and Viktoria for the joy and happiness you bring.

Christina Åkerlind

Linköping, March 2015

\footnotetext{
${ }^{3}$ Rock, Paper, Scissors or for some more familiar as sten, sax, påse.
} 


\section{On(e) Reflection}

What's the color of light?

The sunlight is often said to be white,

but I'm not really sure yet, no, not quite

I know of a prism there was but who, can from it distinguish the red from the blue only by letting the sunlight pass through?

Can you? 


\title{
Contents
}

\author{
Abstract \\ Preface \\ Acknowledgements \\ On(e) Reflection \\ Contents \\ List of Publications
}

1 Introduction 3

1.1 Background 3

1.2 Thesis Outline 5

$2 \quad$ Light and Material Interaction $\quad 7$

2.1 Optical Properties 7

2.2 Optical Modeling 10

3 Polarization in Optics 13

4 Measurement Techniques 17

4.1 Reflection Spectroscopy 17

4.2 Scatterometric Measurements 17

4.3 Ellipsometry 18

4.4 Vector Network Analysis 19

5 Publications $\quad 21$

5.1 Review of Materials for Camouflage 21

5.2 Multispectral Properties 22

5.3 Dynamic Properties and Materials 22

5.4 Natural Materials 23

5.5 Spectral Design 23

5.6 List of Publications and my Contribution $\quad 24$

6 Outlook 25

$\begin{array}{ll}\text { Bibliography } & 27\end{array}$

Papers 


\section{List of Publications}

\section{Paper I}

A review of materials for spectral design coatings in signature management applications, K.E. Andersson and C. Åkerlind, Proc. of SPIE 84 92530Y 1-20 (2014)

\section{Paper II}

Spectroscopic ellipsometry and vector network analysis for determination of the electromagnetic response in two wavelength regions, C. Åkerlind, A. Jänis, H. Kariis, H. Arwin and K. Järrendahl, phys. stat. sol. (c) 5 10889-1092 (2008)

\section{Paper III}

Optical properties and switching of a Rose Bengal derivate: A spectroscopic ellipsometry study, C. Åkerlind, H. Arwin, F.L.E. Jakobsson, H. Kariis and K. Järrendahl, Thin Solid Films 519 5382-3586 (2011)

\section{Paper IV}

Polymer based devices with adaptable infrared refection and transmission, C. Nilsson, E.H. Karlsson and H. Kariis, Proc. of SPIE 6192 61921U 1-8 (2006)

\section{Paper V}

Scattering and Polarization Properties of the Scarab Beetle Chypochilus insulanus cuticle, C. Åkerlind, H. Arwin, T. Hallberg, J. Landin, J. Gustafsson, H. Kariis and K. Järrendahl, Manuscript submitted to Applied Optics (2015) 


\section{Chapter 1}

\section{Introduction}

The purpose of this work is to better understand materials and their optical properties for potential use in spectral design of camouflage. Six evaluation criteria are presented.

\subsection{Background}

Camouflage is an essential property in nature just as it is in defence. The purpose of using camouflage is to hamper detection and thereby the chances to survive a critical situation. This is accomplished by aspiring to look like the surrounding, by having a low contrast towards the background [1]. However, the effectiveness of a camouflage material in a certain situation must be set in relation to the sensitivity of the eyes of the predator or of the sensors of the enemy, as well as to the geometrical orientation between the sun, sensors, background and the camouflaged object.

Colors are an essential part of our daily lives. The physics of color is based on frequency dependent optical effects due to interaction between radiation and materia. Different eyes see differently, due to the variations in the eye construction. Our sensitivity to colors is a highly subjective experience as color perception is based on the sensor ability of the human eye and the signal processing of the brain. To measure and describe color experience, often the CIE $L^{*} a^{*} b^{*}$ color space system [2] is used. The system is based on color representing coordinates for lightness $\left(\mathrm{L}^{*}\right)$, degree of red or green $\left(+/-\mathrm{a}^{*}\right)$ and degree of yellow or blue $(+/-$ $\left.\mathrm{b}^{*}\right)$, respectively. The human eye has receptors for only red, green and blue color. By superposition of the signals from the three receptors almost any color can be perceived. An example is white light that is a combination of many visible wavelengths [3]. The term color can only be related to in the visible part of the spectrum (approximately 400-700 nm), but the effects of interaction between light and materia is not limited to this range. There are individual variations among humans, and even larger differences among species. Dogs have limited color vision, 
some insects can see ultraviolet (UV) light, some snakes are sensitive to infrared (IR), and some birds and beetles have vision sensitive to optical polarization [4]. Artificial receptors, here called sensors, also have a wavelength dependent sensitivity. Polarization sensitive sensors are common in radar range (RR) applications, but still rare in IR. It is however suggested that they can be used to enhance contrasts, once they become available. Military camouflage is therefore required in the whole wavelength range where sensors are common and also against detection of polarization contrasts.

Figure 1.1 shows a ladder model, describing how spectral design is related to military utility. The topic of this thesis lingers at the bottom steps and deal with characterization of optical material properties and surface structural properties. Spectral design, is a method where measuring and modelling material optical properties are prerequisites to design a surface with desired optical response, further explained in Paper I. Spectral design in practice, and the higher steps of the ladder model, are not part of this thesis, but is intensely studied elsewhere.

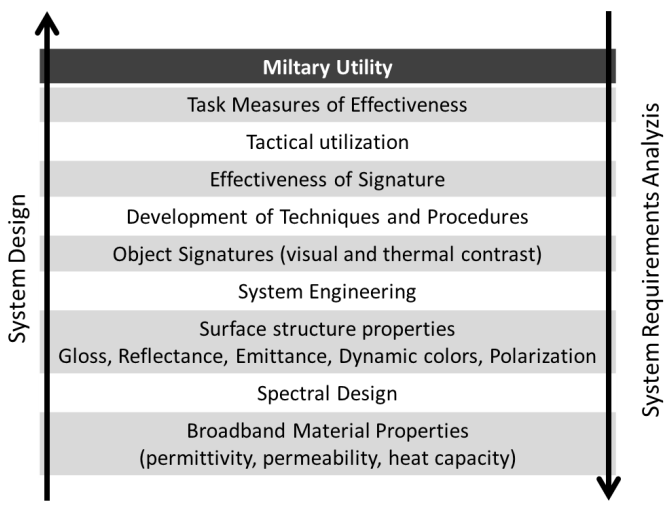

Figure 1.1. A ladder model for helping understanding the system levels between spectral design and military utility.

Six camouflage evaluation criteria related to spectral design (see second step in fig. 1.1) are stated in Paper I. These criteria (in italic below) are to a greater or lesser degree related to the interaction between light and materia and are used in exemplifying issues to consider in the development of VIS-IR-camouflage. Almost all of them will be found in this thesis:

-How surface properties relate to color or wavelength (Reflectance).

-How characteristics of the reflected and emitted radiation depend on electric field orientation (Polarization).

-How a specific background is matched to in real time (Dynamic coloring). -How obstruction between optimized optical properties and optimized microwave properties are avoided (Broadband Properties). 
-How surface structure leads to a matte (diffuse) or a shiny (specular) appearance (Gloss).

-How a temperature contrast in IR is avoided (Emissivity).

In this thesis the camouflage properties in the VIS and IR are in focus. The corresponding electromagnetic radiation in the optical (VIS-IR) range, will be referred to as "light" below. Many of the fundamental properties are however the same for other wavelength regions.

There are also other needs to be fulfilled for an efficient and usable camouflage (surface adhesion, mechanical robustness, power supply, self-cleaning, etc.). Those are of high interest but beyond the scope of this work.

\subsection{Thesis Outline}

After the introduction to the thesis, given in section 1.1 above, chapter 2 introduces different aspects of interaction between light and materia. In chapter 3, a description of optical polarization properties are presented. Experimental methods are presented in chapter 4 . In chapter 5 , the papers are summarized, and examples of future research are given in chapter 6 . A thorough explanation of the need of materials for camouflage application and military utility of spectral design is found in Paper I [5]. Broadband studies of the optical properties of different materials are found in Paper II [6]. Studies of dynamic properties of a dye are presented in Paper III [7] and of a polymer in Paper IV [8]. Scattering and polarization properties of a natural structure are presented in Paper $\mathrm{V}$ [9]. 


\section{Chapter 2}

\section{Light and Material Interaction}

What happens when light interacts with a material and how do we describe this interaction?

\subsection{Optical Properties}

When light interacts with a material, it is reflected, absorbed or transmitted, as illustrated in fig. 2.1. The interaction depend on the properties of the light and the

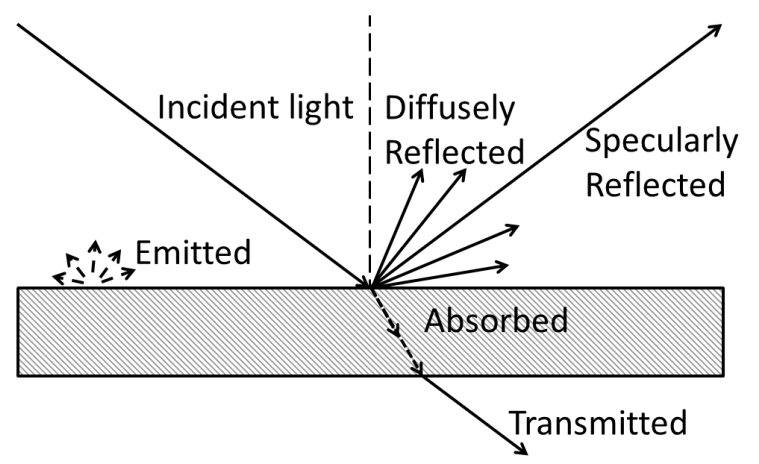

Figure 2.1. Light can be reflected, absorbed and transmitted.

material at the wavelengths involved. Described through Maxwell's equations [10], a unified theory valid for any system at any wavelength $\lambda$ is present. The equations relate the field or wave properties of light to different material specific properties, 
$\epsilon, \mu$ and $\sigma$, the dielectric function, (or the relative permittivity), the relative permeability and the conductivity of the medium, respectively. In the description below, the conductivity is included in the dielectric function.

To describe optical properties of materials, the complex-valued refractive index $N$ is useful. $N, \epsilon$ and $\mu$ are generally related through $N=\sqrt{\epsilon \mu}$, where $\epsilon=\epsilon_{1}+i \epsilon_{2}$ is a complex entity and central for describing material optical properties. The real and imaginary parts $\epsilon_{1}$ and $\epsilon_{2}$ are not independent, but related through the Kramers-Kronig relation [11]. $N$ is in general also complex and expressed as $N=n+i k$, where $n$ is the real part describing refraction and $k$ the imaginary part, also called the extinction coefficient. The parameter $k$ is related to the absorption coefficient $\alpha=4 \pi k / \lambda$. Various absorption processes are related to the work in this thesis. An example is the absorption bands of the atmosphere in the infrared region constraining the useability of sensors to specific wavelength bands, generally to 3-5 and 8-12 $\mu \mathrm{m}$. Another example is related to vibration modes in chemical bonds between atoms and molecules, when energy is absorbed or emitted. The vibration modes give rise to characteristic absorption resonances and are similar for compounds with similar chemical compositions [12]. The combination of absorption bands give each compound a specific chemical "fingerprint". An absorption band spectrum can be revealed through for example spectroscopic reflectance measurements. Examples of identified absorption resonance peaks are reported in the included Papers II, III and V.

Specular reflection from a smooth (glossy) surface, such as a mirror, are described by Fresnel's ${ }^{1}$ reflection equations, 2.1 [13].

$$
\begin{aligned}
& r_{p}=\left(E_{0 r p} / E_{0 i p}\right)=\left(n_{t} \cos \theta_{i}-n_{i} \cos \theta_{t}\right) /\left(n_{t} \cos \theta_{i}+n_{i} \cos \theta_{t}\right) \\
& r_{s}=\left(E_{0 r s} / E_{0 i s}\right)=\left(n_{i} \cos \theta_{i}-n_{t} \cos \theta_{t}\right) /\left(n_{i} \cos \theta_{i}+n_{t} \cos \theta_{t}\right)
\end{aligned}
$$

Here $E_{0 i}$ and $E_{0 r}$ are the amplitudes of the incident and reflected fields at the boundary, and $n_{i}$ and $n_{t}$ the refractive indexes as shown in fig. 2.2. The incident angle $\theta_{i}$ is then the same as the angle of reflection $\theta_{r}$, and $\theta_{t}$ is the angle of refraction. A light wave travelling in one direction, can be expressed as two orthogonal components. In the case of a reflection with an incoming, reflected and transmitted wave it is convenient to choose one component of the E-field to be parallel to the plane of incidence (denoted with index p) ${ }^{2}$ and one component to be perpendicular (denoted with index s) ${ }^{3}$ to the plane of incidence. This situation is shown in fig. 2.2 where the plane of incidence is the same as the plane of the paper. The differences in the reflection properties of the two components, the reflection coefficients $r_{p}$ and $r_{s}$, are described in eq. 2.1. The reflectance $R$, defined as the ratio of the reflected power (or flux) to the incident power (flux), can be expressed as $R=I_{r} / I_{i}$, the ratio between the reflected and incident irradiance. Since the irradiance is proportional to the square of the field amplitude $E_{0}$,

\footnotetext{
${ }^{1} \mu=1$ is assumed

$2 \mathrm{p}$ from the German word parallel for parallel.

$3 \mathrm{~s}$ from the German word senkrecht for perpendicular.
} 
the reflectance $R$ of the two orthogonal components are related to the Fresnel's reflection coefficients according to,

$$
\begin{aligned}
& R_{p}=\left|r_{p}\right|^{2}=\left(\left|E_{0 r p}\right| /\left|E_{0 i p}\right|\right)^{2} \\
& R_{s}=\left|r_{s}\right|^{2}=\left(\left|E_{0 r s}\right| /\left|E_{0 i s}\right|\right)^{2}
\end{aligned}
$$

The ratio between the incident and transmitted irradiance can in a similar way be described with the transmittance $T$. A surface that is not smooth or glossy reflect also in other directions than the specular. It is then referred to as diffuse reflection or scattering, see fig. 2.1. Depending on the wavelength of the light, a surface can be smooth in some cases but seem rough in others. These kind of properties are discussed in Paper V.

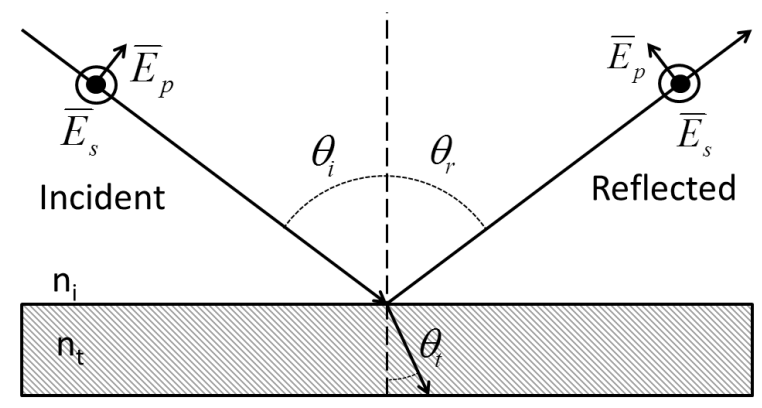

Figure 2.2. The reflection of a plane wave on a surface. The $p$-polarized light $E_{p}$ is oscillating in the plane of the paper, and the $s$-polarized light $E_{s}$ is oscillating perpendicular to the plane of the paper. $\theta_{i}=\theta_{r}$ in specular reflection.

Any radiation not reflected or transmitted is absorbed and can be specified with the absorptance $A$. Conservation of energy leads to the relation $R+T+A=1$. The emissivity $\varepsilon_{m}$ is an optical property of a material containing information about the thermal radiative behavior, i.e. the ability of the material to emit energy. Under geometrical and spectral averaging the emissivity and absorptance is equal and for certain conditions [14], $e_{m}=A$. The more reflective an opaque material is, the lower is the emissivity. Emissivity is angle and wavelength dependent, and can be expressed as the ratio between the energy emitted from an object or material ${ }^{4}$ $\left(\varepsilon_{m}<1\right)$, to the energy emitted by a perfect blackbody $\left(\varepsilon_{m}=1\right)$ at the same temperature. All objects or bodies emit blackbody radiance according to StefanBoltzmann's law [15], due to its temperature. A body of the temperature $20^{\circ} \mathrm{C}$ will emit radiance in the IR with a peak value at $10 \mu \mathrm{m}$. At this wavelength the atmosphere is highly transmitting and IR sensors can be used at long distances which means that the detectably is high.

\footnotetext{
4 the emittance
} 
When light is diffusely reflected or scattered, the light is spread in many directions, for instance when the sunlight is scattered in the atmosphere by the large amount of particles in the air or when a beam is reflected by a rough surface. The wavelength, the roughness, or size and concentration of particles are of major importance for the type of scattering that occur. Usually scattering is divided into three categories determined by the structure size $(\varnothing)$ compared to the wavelength $(\lambda)$.

Rayleigh scattering $(\varnothing<\lambda)$ :

When the light is interfering with particles or structures of dimensions smaller than the wavelengths the scattering is called Rayleigh scattering. [3] This can be exemplified by the atmosphere scattering sunlight, whereby the sky is diffusely radiating. Rayleigh scattering is frequency dependent, and short wavelengths (blue light) are scattered more efficiently, explaining the color of the sky.

Mie scattering $(\varnothing=\lambda)$ :

When the particles are of similar size as the interacting wavelengths, the phenomenon is called Mie scattering [3]. Visible light scattered according to Mie in a cloud of water droplets, causes the cloud appear white.

$\varnothing>\lambda$ :

When light interacts with particles or material of dimensions larger than the wavelengths $(\varnothing>\lambda)$, other more complex models are needed.

An important concept in the scattering context is the Lambertian surface. This type of surface is ideal diffusely reflecting, scattering the light equally in all directions and fulfills Lambert's cosine law,

$$
I_{\theta}=I_{n} \cos \theta
$$

stating that the irradiance $I_{\theta}$, the angle $\theta$ from the surface normal, is proportional to the $\cos \theta$ and the irradiance in the normal direction $I_{n}$. However, the Lambertian properties are wavelength dependent. A surface scattering infrared wavelengths according to the Mie theory might scatter microwaves according to the Rayleigh theory. A surface Lambertian in the visible is not necessary Lambertian for other wavelengths. Lambertian properties are discussed in Paper V.

\subsection{Optical Modeling}

In many cases there is a need to use theoretical models of optical properties. A basic view of atom and molecule bonds is the starting point for the Lorentz oscillator model. A covalent bond between two nuclei $m_{1}$ and $m_{2}$ is a combination of an attraction, that increases when the nuclei are parted, and a repulsive force. Often it is useful to look at the bond as a mass-spring system. If disturbed it may vibrate with a frequency $\omega$ determined by the spring constant $k_{s}$, and the effective mass $m$ of the bodies, $m_{1}, m_{2}$ as depicted in the inset in fig. 2.3. 


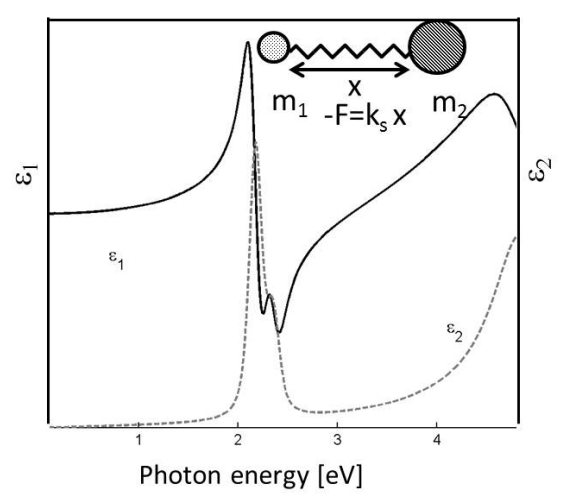

Figure 2.3. Illustration of the relation between $\epsilon_{1}$ and $\epsilon_{2}$ of a combination of a Lorentzian resonance. The Lorentz expression is based on the classic spring model (inserted).

If a harmonic electromagnetic field $\mathbf{E}(\omega)$ is the driving force behind movements within a material, like in the spring above, there will at some frequency $\omega$ be a resonance in the system. This happens at the angular frequency $\omega_{0}$, and can be described by the Lorentz expression,

$$
E(\omega)=1-\frac{\omega_{p}^{2}}{\omega^{2}-\omega_{0}^{2}+i \omega / \tau}
$$

Here, $\omega_{p}$ is the resonance frequency of all contributing electrons, (the plasma frequency) and $\tau$ is the lifetime related to the resonance broadening. A simple free electron model, the Drude model ${ }^{5}$ is obtained if we let $\omega_{0}=0$ in eq. 2.4. The spring system model or Lorentz oscillator [3] model, describes the absorption resonance peak(s) observed for dielectric and semiconductor materials (see fig. 2.3). When modeling a more complex sample with many layers, different models characteristics, such as the Lorentz oscillators, are used together with reference data to solve for unknown optical and structural parameters of the sample. The modelling process is iterative, where model parameters are matched to experimental data and the mean square error (MSE) can be used as a measure of the goodness of fit [16]. The Lorentz expression was used when determining the dielectric function $\epsilon$ and the film thicknesses in the dynamic color studies of Rose Bengal, reported in Paper III.

\footnotetext{
${ }^{5}$ Drude developed the theory of ellipsometry.
} 


\section{Chapter 3}

\section{Polarization in Optics}

A brief introduction to optical polarization and its description is given in this chapter.

If light propagates in the $z$-direction the electric field of a plane wave $\mathbf{E}(z, t)$ can be described as,

$$
\begin{aligned}
\mathbf{E}(z, t) & =\mathbf{E}_{x}(z, t)+\mathbf{E}_{y}(z, t) \\
& =E_{x}(t) \cos \left(q z-\omega t+\delta_{x}(t)\right) \hat{\mathbf{x}}+E_{y}(t) \cos \left(q z-\omega t+\delta_{y}(t)\right) \hat{\mathbf{y}}
\end{aligned}
$$

where the total $\mathbf{E}$-field consist of the components $\mathbf{E}_{x}(z, t)$ and $\mathbf{E}_{y}(z, t)$, which have a phase difference $\delta(t)=\delta(x)-\delta(y) . E_{x}(t)$ and $E_{y}(t)$ are the component amplitudes and $q$ is the wave vector.

Polarization is a wave property that describes the orientation of an electric field vector in relation to the propagation direction of an $\mathbf{E}$-field. The case when $\mathbf{E}_{x}(z, t)$ and $\mathbf{E}_{y}(z, t)$ are completely correlated, is defined as totally polarized light. If $\mathbf{E}_{y}(z, t)=0$, the light is linearly polarized in the x-direction. With $\mathrm{x}$-and $\mathrm{y}$ components of equal amplitude and phase, the resulting $\mathbf{E}$-field will be linearly polarized $+45^{\circ}$ related to the $\mathrm{x}$-axis. If the phase difference between the $\mathrm{x}$ - and $\mathrm{y}$ - components is $\pm 180^{\circ}$ (half a wavelength) the light is linearly polarized $-45^{\circ}$ in relation to the $\mathrm{x}$-axis.

In the case of circular polarization, the amplitude is the same for the $\mathrm{x}$ - and $y$-directions, and the phase difference is $\pm 90^{\circ}$ (a quarter of a wavelength). The described polarization states are presented in fig. 3.1.

In this work the following convention is used. A wave oscillating clockwise while propagation towards you is considered having right-handed polarization, while counterclockwise oscillation propagation towards you is refereed to as left-handed polarization. Any other phase difference than exemplified above will result in elliptic polarization. 
The two components of polarized light, in the $\mathrm{x}$ and $\mathrm{y}$ direction, respectively, have different reflection properties. An example of this is the Brewster angle (polarization angle) $\theta_{i}=\arctan \left(n_{t} / n_{i}\right)$ for which only the polarized part perpendicular to the plane of incidence (x-component) is reflected. In reflection contexts, the notation s- and p-polarization is a common description of the perpendicular and parallel polarization components, as exemplified in chapters 2 and 4 .
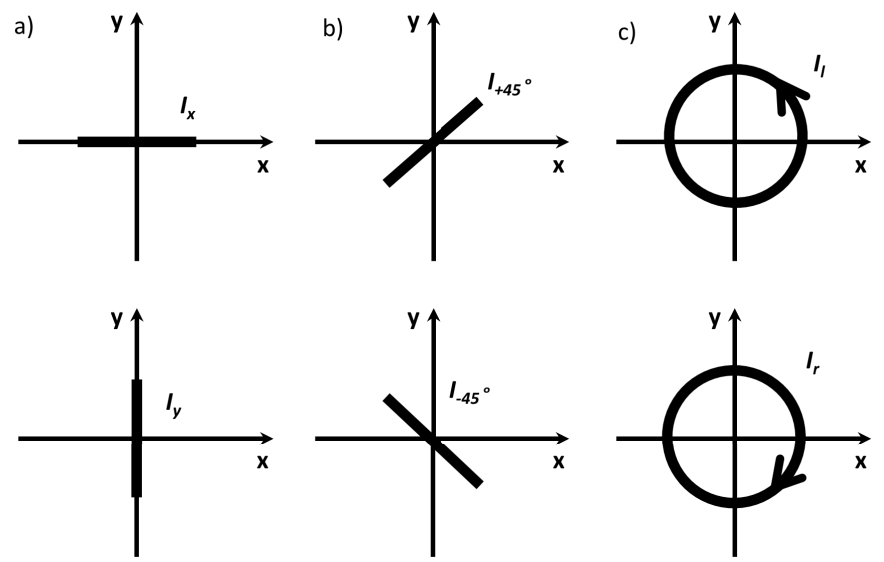

Figure 3.1. A schematic description of different polarization states of light when looking into the source of light. a, and b) Linear polarization (in different directions). c) Leftand right-handed circular polarization.

The use of the polarization ellipse (presented in fig. 3.2) can be helpful when describing polarization states. Through the characteristics of the polarization ellipse the fundamental polarization properties can be expressed [13] using the azimuth angle $\alpha(-\pi / 2 \leq \alpha<\pi / 2)$ which defines the orientation of the major axis a of the ellipse in its plane, and the ellipticity $e$, which is defined as $e=b / a$, i.e. the fraction between the minor and major axis $b$ and $a$, of the polarization ellipse. The ellipticity e describes to which extent the reflected light is circular or linearly polarized. These parameters have been used in Paper V to describe the polarization properties of a scarab beetle. When the light is partly polarized there is a need to describe the degree of polarization $P$. It can be expressed through the fraction between the irradiance of the polarized part of the wave, $I_{p o l}$, and the total irradiance $I_{t o t}$,

$$
P=\frac{I_{p o l}}{I_{t o t}}=\frac{\sqrt{\left(Q^{2}+U^{2}+V^{2}\right)}}{I}
$$

The Stokes formalism is suitable for description of the polarization of light and the Stokes elements $I, Q, U, V$ can be used for describing the mixture of polarized and unpolarized light. The Stokes elements $I, Q, U, V$ are expressed as different combinations of the irradiance and degree of polarization as in eq. 3.3- 3.6, where $<>$ represent the time averaged value and $\mathbf{E}_{x}(t), \mathbf{E}_{y}(t), \delta_{x}(t)$ and $\delta_{y}(t)$ are defined as in eq. 3.1. 


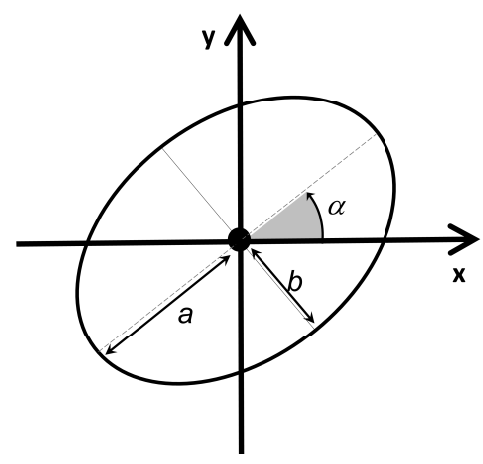

Figure 3.2. The polarization ellipse with a description of the azimuth angle $\alpha$ and the ellipticity e defined as $e=b / a$.

$$
\begin{aligned}
I=I_{x}+I_{y} & =\frac{n}{2} \sqrt{\frac{\epsilon_{0}}{\mu_{0}}}<E_{x}^{2}(t)>+<E_{y}^{2}(t)> \\
Q=I_{x}-I_{y} & =\frac{n}{2} \sqrt{\frac{\epsilon_{0}}{\mu_{0}}}<E_{x}^{2}(t)>-<E_{y}^{2}(t)> \\
U=I_{+45^{\circ}}-I_{-45^{\circ}} & =n \sqrt{\frac{\epsilon_{0}}{\mu_{0}}}<E_{x}(t) E_{y}(t) \cos \left(\delta_{y}(t)-\delta_{x}(t)\right)> \\
V=I_{r}-I_{l} & =n \sqrt{\frac{\epsilon_{0}}{\mu_{0}}}<E_{x}(t) E_{y}(t) \sin \left(\delta_{y}(t)-\delta_{x}(t)\right)>
\end{aligned}
$$

Often the incoming Stokes vector $\mathbf{S}_{\mathbf{i}}$ is normalized by setting $I_{i}=1$. The $Q$ and $U$ elements represent linearly polarized irradiance with preference for $\mathrm{x}$ or $\mathrm{y},+45$ or -45 , respectively. $V$ is the right- or left-handed circular polarization preference. Through the so called Mueller matrix [17] with 16 elements $M_{i j}$, the outgoing Stokes vector $\mathbf{S}_{\mathbf{o}}$ is related to the incoming Stokes vector $\mathbf{S}_{\mathbf{i}}$,

$$
\mathbf{S}_{\mathbf{o}}=\left[\begin{array}{c}
I_{o} \\
Q_{o} \\
U_{o} \\
V_{o}
\end{array}\right]=\left[\begin{array}{llll}
M_{11} & M_{12} & M_{13} & M_{14} \\
M_{21} & M_{22} & M_{23} & M_{24} \\
M_{31} & M_{32} & M_{33} & M_{34} \\
M_{41} & M_{42} & M_{43} & M_{44}
\end{array}\right]\left[\begin{array}{c}
I_{i} \\
Q_{i} \\
U_{i} \\
V_{i}
\end{array}\right]=\mathbf{M S}_{\mathbf{i}}
$$

The matrix represent an optical system, where the elements $M_{i j}$ are different combinations of reflection or transmission coefficients. Often the Mueller matrix is normalized so that $M_{11}=1$. The interaction between light and materia can be fully described using the Mueller matrix in eq. 3.7. If a sample can be described using a Jones matrix [18], it can also be described with a Mueller matrix. Since Jones matrices do not describe partial polarization, the opposite is not true.

From the Mueller-matrix elements $M_{i j}$, the degree of polarization $P$ can be calculated for different source polarization states, using the Stokes vector. This was done in the study of the scarab beetle in Paper V. 


\section{Chapter 4}

\section{Measurement Techniques}

This chapter briefly describes the main characterization techniques employed in this thesis.

\subsection{Reflection Spectroscopy}

To study reflection and its wavelength dependent properties, a spectrometer can be used. When combined with an integrating sphere, not only specular but also diffuse or scattering properties can be measured. Separate instruments are often used in the UV-VIS-NIR region and the IR region. For shorter wavelengths it is common to employ instruments measuring the wavelengths one by one, i.e. dispersive instruments. Using the Fourier Transform InfraRed (FTIR) spectrometric technique, the material surface is instead illuminated using all wavelengths simultaneously. The signal is then Fourier transformed to receive the reflectance versus wavelength. To determine the spectral directional hemispherical reflectance (SDHR), the setup described in fig. 4.1 can be used, allowing measurement of the total (specular and diffuse) reflectance. The sample is directly illuminated, and the detector is collecting the light scattered from the sample via the surface of the sphere within the detector field of view. Often the reflectance measurements are performed close to normal incidence to minimize the impact of the polarization properties of the incoming beam. Reflectance standards with diffuse and specular surfaces are used for calibration. The reflectance spectrum can also reveal material specific absorption resonances.

\subsection{Scatterometric Measurements}

A bidirectional reflection distribution function (BRDF) instrument can be used to investigate angle dependent scattering properties. With this technique it is 

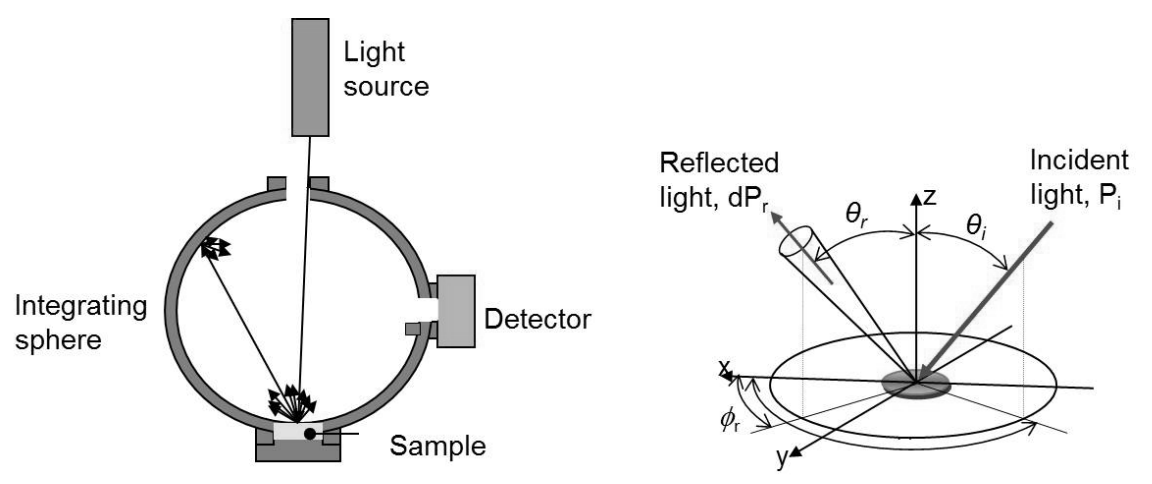

Figure 4.1. A schematic view of the SDHR (left) and BRDF (right) measurement setup.

possible to determine how incident light is reflected in space by measurements of all angles in the hemisphere. The bidirectional reflection distribution function $f_{B R D}$ is defined as the fraction between the differential radiance and differential irradiance and is measured in inverse steradians.

$$
f_{B R D} \cong \frac{d P_{r}}{d \Omega_{r} P_{i} \cos \theta_{r}} \cong \frac{P_{r} / \Omega_{r}}{P_{i} \cos \theta_{r}}[1 / s r]
$$

$f_{B R D}$ can be expressed by the incident power from the light source $\left(P_{i}\right)$, the scattered power reaching the detector $\left(P_{r}\right)$ and, the geometry of the reflected scatter, where the solid angle $\Omega_{r}$ is determined by the detector aperture area $A$ and the distance $r$ between the sample and detector $\left(\Omega_{r}=A / r^{2}\right) . \cos \theta_{r}$ is a correcting factor to adjust for the illuminated area to its size viewed from the scatter direction, $\theta_{r}$. The bi-directionality of the $f_{B R D}$ is dependent on the incident direction and the scatter direction, (fig. 4.1), and may be viewed as the directional reflectance per unit solid angle of collected scattered radiation [19].

\subsection{Ellipsometry}

Ellipsometry is based on the analysis of change in polarization for a reflected beam compared to the incoming beam. Figure 4.2 describes schematically an ellipsometry instrument setup; the light path from the source, through a polarizer and a compensator before reflected in the sample, and through a compensator and an analyzer (polarizer) before collected at the detector. The outcome of an ellipsometric measurement is expressed through the complex ellipsometric parameter $\rho$, the ratio between the (complex valued) Fresnel's reflection coefficients $r_{p}$ and $r_{s}$ of the measured material for p-polarized light and s-polarized light, (eq. 2.1). Using the ellipsometric angles $\Psi$ and $\Delta,[13] \rho$ can be written on polar form,

$$
\rho=r_{p} / r_{s}=\tan \Psi \cdot e^{i \Delta}
$$


From $\rho$, sample optical properties such as the dielectric function $\epsilon$ can be determined. In the general case the so called the pseudo-dielectric function, is obtained through inversion of the equation

$$
<\epsilon>=\sin ^{2} \theta\left[1+\frac{(1-\rho)^{2}}{(1+\rho)^{2}} \tan ^{2} \theta\right]
$$

In the case of measurements on an ambient-bulk system the dielectric function is obtained directly from eq. 4.3 (as in Paper II). However, in most cases the sample contains one or more layers including interface and surface roughness and direct useful information on optical and structural properties is not obtained without additional modelling. In this sense ellipsometry is well suited for analysis of surfaces, thin films and heterostructures. Since the technique relies on phase information and amplitude ratios the measurements become very sensitive. When there is no

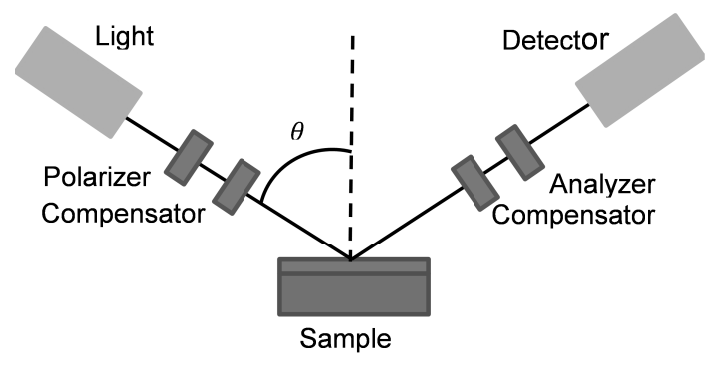

Figure 4.2. A schematic view of an ellipsometric measurement setup.

coupling between the orthogonal polarization components, standard ellipsometry can be used. For analysis of anisotropic and/or depolarizing samples, Mueller Matrix Spectroscopic Ellipsometry (MMSE) is a more powerful technique and is required to describe depolarization. A MMSE setup proposed by Azzam, [20] provides the opportunity for multiple incident polarization states (linear, circular and elliptic) of the light and has been realized in dual-rotating-compensator ellipsometers. The light illuminating the sample in a MMSE setup, is changed to take a set of different polarization states (different Stokes vectors). This allows analysis of the reflected Stokes vector $\mathbf{S}_{\mathbf{o}}$, and all the 16 elements of the Mueller matrix can be determined from the relation $\mathbf{S}_{\mathbf{o}}=\mathbf{M} \mathbf{S}_{\mathbf{i}}$ according to eq. 3.7. The output data is often used for modeling of sample properties. This was done in Paper III. MMSE was also used to obtain polarization properties of a depolarizing surface found in the cuticle of the scarab beetle (Paper V).

\subsection{Vector Network Analysis}

Maxwell's equations are valid for all wavelengths. Materials however, react differently depending on the wavelength of the interacting waves. For some wavelengths or frequencies, it is more useful to use circuit theory with resistances and 
impedances ( $Z$ and $Z_{0}$ in fig. 4.3). Using a Vector Network Analyzer (VNA) in the microwave range, the change in amplitude and phase for the reflected $\mathbf{S}_{\mathbf{1 1}}$ and transmitted $\mathbf{S}_{\mathbf{2 1}}$ beam can be determined through two-port measurements. In such a measurement two parts of waveguide constrict the sample with the sample holder as an integral part of the waveguide (fig. 4.3). The measurements can be performed using different waveguides in several frequency bands, using one waveguide for each measurement range. Once the reflected $\mathbf{S}_{\mathbf{1 1}}$ and transmitted beam $\mathbf{S}_{\mathbf{2 1}}$ (expressed with the reflection coefficient from Fresnel's equation) have been experimentally determined, the dielectric function $\epsilon$ can be calculated as described in Paper II.

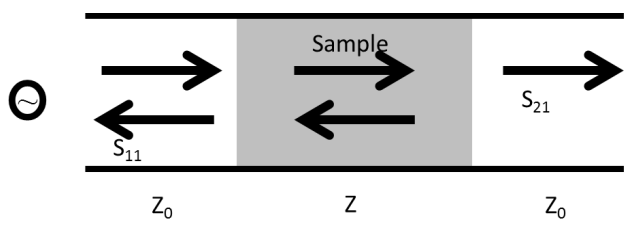

Figure 4.3. Configurations of the VNA measurement setup. $\mathrm{Z}$ is the sample impedance and $Z_{0}$ is the vacuum impedance. 


\section{Chapter 5}

\section{Publications}

In this chapter relevant material categories and phenomena are briefly described together with the studied materials. The chapter also includes a summary of the major results obtained in the included papers.

The material characteristics experimentally studied in this thesis are very different. Samples ranging from semiconductors, dynamic colored thin films, beetle cuticles to doped dielectrics are schematically presented in fig. 5.1. These may not themselves be relevant for camouflage applications, but they help to illustrate and exemplify important issues and phenomena that are. The variety of materials that are interesting for camouflage issues also come clear in the review presented in Paper I.

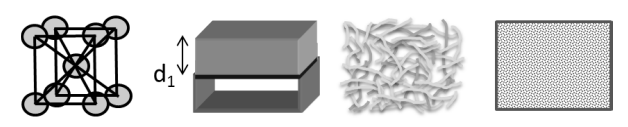

Figure 5.1. Different structures and samples studied in the thesis. From left: crystalline silicon, thin dynamic colored films, inhomogeneously scattering natural cuticle structures and doped epoxy.

\subsection{Review of Materials for Camouflage}

The first paper presents the application field and a review of materials for camouflage. A vast range of materials can be useful for camouflage. The reviewed materials have been classified roughly in order of complexity from single layers of paint, to three dimensional (3D) structures, and into biomimetic structures and metamaterials. These are all promising candidates for camouflage, but have also other areas of application. Six criteria for evaluation of camouflage are suggested. 
Further studies on these can aid demonstration of military utility by spectral design.

Paper I: The major result is a review of materials for signature management development trends and a description of six camouflage evaluation criteria for future use.

\subsection{Multispectral Properties}

Often in optics, surface properties are of great importance. However, the surface ability to reflect, transmit or absorb and the relevance or impact of a roughness layer or structure, is wavelength dependent. Structures with properties optimized for one wavelength range, may have interfering impact on properties in another range, if not properly constructed and optimized in advance. Therefore it is of relevance to study $\epsilon$ in a wide range, as was done in the broadband studies (VISIR; RR) presented in Paper II. Materials were chosen to have different reflection and absorption properties in the different wavelength ranges covered in the study. Epoxy $^{1}$ is transparent for VIS and RR, but when doped with FeCo-nanoparticles it became opaque in VIS and IR. Epoxy when cured is a mechanically hard and temperature stable material with a range of industrial applications. For comparison, studies were performed on an IR transmitting material, silicon (opaque in VIS and RR). Silicon wafers are widely used in industry as substrates for fabrication of electronic or photovoltaic devices, and integrated circuits.

Paper II: The dielectric constant $\epsilon$ studied in a wide range (VIS-IR; RR), using wavelength specific techniques was reported.

\subsection{Dynamic Properties and Materials}

One way of classifying optical controllable dynamic materials with switchable properties, is to distinguish between organic and inorganic materials. Another classification is according to the external stimulus of the dynamic effect. The origin of a color shift can have several causes, depending on the material composition and sensitivity.

In chemically switchable materials, a solvent, ion or $\mathrm{pH}$-index change can be the trigger of color switching. An example of a chemically switchable materials is Rose Bengal $(\mathrm{RB})^{2}$. RB has been intended for use in photovoltaic devices [21] and in bi-stable organic memory devices [22].

Paper III: Thin film thicknesses, and the dielectric constant $\epsilon$ in two different oxidation configurations were reported for the switching dye Rose Bengal.

Other examples are thermo-, piezo-, and electrochromic materials where in the latter case reflectance and/or absorption often is reversibly changed by the application of a voltage.

In electrochromic materials such as conjugated polymers, the origin of dynamic

\footnotetext{
${ }^{1}$ A dielectric polymer (diglycidyl ether bisphenol-A) cured through crosslinking reactions.

2 [4,5,6,7-tetrachloro-2', $4^{\prime}, 5^{\prime}, 7^{\prime}$-tetraiodo-fluorescein]
} 
coloring is explained by changes in the electronic structure of the backbone upon doping and undoping, i.e. reduction and oxidation. The extensively studied conducting polymers PEDOT:PSS ${ }^{3}$ [23, 24], is despite transparent and conducting properties also commonly used for antistatic surface treatments. At the time when Paper IV was written, reports on dynamic IR properties of PEDOT were scarce. A reason was that the outermost layer often is an electrolyte, opaque to IR.

Paper IV: Transmission and reflection properties in IR of PEDOT based electronic devices was reported.

\subsection{Natural Materials}

The complexity of structures in nature is remarkable, compared to the limited choice of elements ( $\mathrm{C}, \mathrm{H}, \mathrm{O}, \mathrm{N})$ for the most common biopolymers; cellulose of plants and trees, and chitin in shell of crustaceans, mollusks, and cuticle of insects. With an increased ability in fabrication of nanostructures there is a renewed interest in studies of structures of biology, for mimic purposes. Examples of interesting study objects are structures with non-bleaching light-weight structural colors [25] or unordered structures of chitin from the cuticle of the beetle Chypochilus insulanus (Moser, 1918) scattering visible light. The C. insulanus, cuticle is covered with thin scales filled with randomly oriented fibrils of chitin [26], causing the beetle appear white, see fig. 5.2.

Paper V (manuscript): Degree of polarization, reflection and scattering properties were reported for the cuticle of the scarab beetle Chypochilus insulanus.

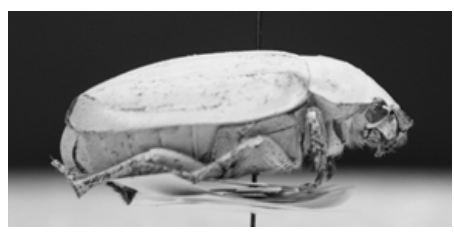

Figure 5.2. The white scarab beetle Chypochilus insulanus.

\subsection{Spectral Design}

One of the first steps working with spectral design is to characterize the materials of interest. This has been performed in the work presented in this thesis. Combining materials, layer-structures and functionality for a given purpose is the core of spectral design. Inspiration from nature and natural structures can together with structural design improve material properties and are of relevance for spectral design and a route to practical applications.

\footnotetext{
${ }^{3}$ [Poly(3,4-ethylenedioxythiophene):Polystyrene sulfonate]
} 


\subsection{List of Publications and my Contribution}

PAPER I [5]

A review of materials for spectral design coatings in signature management applications,

K.E. Andersson and C. Åkerlind,

Proc. of SPIE 84 92530Y 1-20 (2014)

My contribution to this paper is discussions about content and part of the writing.

PAPER II [6]

Spectroscopic ellipsometry and vector network analysis for determination of the electromagnetic response in two wavelength regions,

C. Åkerlind, A. Jänis, H. Kariis, H. Arwin and K. Järrendahl,

phys. stat. sol. (c) 5 10889-1092 (2008)

My contribution to this paper is the optical measurements and part of the writing.

PAPER III [7]

Optical properties and switching of a Rose Bengal derivate: a spectroscopic ellipsometry study,

C. Åkerlind, H. Arwin, F.L.E. Jakobsson, H. Kariis and K. Järrendahl,

Thin Solid Films 519 5382-3586 (2011)

My contribution to this paper is part of the measurements and most of the writing.

PAPER IV [8]

Polymer based devices with adaptable infrared reflection and transmission,

C. Nilsson, E.H. Karlsson and H. Kariis,

Proc. of SPIE 619261921 U 1-8 (2006)

My contribution to this paper is part of measurements and most of the writing.

PAPER V [9]

Scattering and Polarization Properties of the Scarab Beetle Chypochilus insulanus cuticle,

C. Åkerlind, H. Arwin, T. Hallberg, J. Landin, J. Gustafsson, H. Kariis and K. Järrendahl,

Manuscript submitted to Applied Optics 2015

My contribution to this paper is part of the measurements and most of the writing. 


\section{Chapter 6}

\section{Outlook}

Here direction and areas for future research of the field spectral design of materials for camouflage and signature management are suggested.

The recent improvements in material science and nano-fabrication technology show promising and inspiring development for future effective camouflage. The future direction has several aspects to consider, the academic approach must be tempered with the military utility and requirements. With aid of the ladder model fig. 1.1(see Paper I) demonstration of military utility of spectral design is benefitted and related to what is written below. Issues to consider for future spectral design are for example:

- Methodology to formulate technical requirements from information on scenario and background.

- Choice of scenario and background to demonstrate methodology and apply requirements.

- Evaluation of material and modelling trends and their potential for camouflage applications.

- Methodology for choice of complexity and combination of materials and structures.

- Performance of spectral modelling and putting requirements on the design.

- Studying how to realise spectral design through different fabrication methods.

- Fabrication and realization of chosen structure and advanced material properties.

- Measurement and evaluation of results. 
- Incorporation of additional functionalities such as self-cleaning properties and mechanical robustness.

- Studying the balance between signature managements and other types of electronic warfare.

My personal wish for future work stands on three legs:

Polarization properties. These are not thoroughly studied in the signature management context for optical wavelengths, although it has been used for long in radar applications. Future research ought to cover materials with specific polarization properties.

Biomimetics is an interesting area in itself, but also relevant for future studies [27]. Diffuse "white" scattering surfaces with Lambertian properties in the IR region are interesting. Could the structures of the beetle C. insulanus (Paper V) be scaled up to receive the wanted optical properties in longer wavelengths?

Metamaterials, where structures of dimensions smaller than the wavelength is the origin of novel macroscopic behavior, are predicted to have a "brilliant future" [28]. Metamaterials might in the future give possibility of invisibility of an object, rather than adapting to the background. This could take camouflage technology to new levels. 


\section{Bibliography}

[1] G. Hartcup. Camouflage A History of Concealment and Deception in War. Pen \& Sword Military, 2008.

[2] Cie technical report, colorimetry. Technical Report 3d ed. draft CIE, International Commission on Illumination (French Commission internationale de l'éclairage), 2004.

[3] E. Hecht. Optics. Addison-Wesley, 2002.

[4] G. Horváth and D. Varjú. Polarized light in Animal vision, Polarization patterns in nature. Springer, 2003.

[5] C. Åkerlind and K.E. Andersson. A review of materials for spectral design coatings in signature management applications. Proc. of SPIE, 84(92530Y):120, 2014.

[6] C. Åkerlind, A. Jänis, H. Kariis, H. Arwin, and K. Järrendahl. Spectroscopic ellipsometry and vector network analysis for determination of the electromagnetic response in two wavelength regions. physica status solidi (c), 5(5):10891092, 2008.

[7] C. Åkerlind, H. Arwin, F.L.E. Jakobsson, H. Kariis, and K. Järrendahl. Optical properties and switching of a Rose Bengal derivate: A spectroscopic ellipsometry study. Thin Solid Films, 519:5382-3586, 2011.

[8] C. Nilsson, E.H. Karlsson, and H. Kariis. Polymer based devices with adaptable infrared reflection and transmission. Proc.of SPIE, 6192(61921U).

[9] C. Åkerlind, H. Arwin, T. Hallberg, J. Landin, J. Gustafsson, H. Kariis, and K. Järrendahl. Scattering and polarization properties of the scarab beetle Chyphochilus insulanus cuticle. submitted to Applied Optics 2015.

[10] M. Born and E. Wolf. Principles of Optics. Cambridge University Press, 9th edition, 2013.

[11] F. Wooten. Optical Properties of Solids. Academic Press, Inc., 1972. 
[12] D.A. Burns and E.W. Ciurczak. Handbook of Near-Infrared Analysis, volume 13 of Practical Spectroscopy Series. Marcel Dekker, Inc., 1992.

[13] R.M.A. Azzam and N.M. Bashara. Ellipsometry and Polarized Light. Elsevier Science Publisher, 1977.

[14] J.M. Palmer. The measurement of transmission, absorption, emission, and reflection, in: M. Bass,(Ed.). Handbook of Optics, volume II Devices, Measurements, and Properties. McGraw-Hill, Inc., 1995.

[15] K. Krane. Modern Physics. John Wiley \& Sons, 1983.

[16] J.A. Woollam. Ellipsometry, variable angle spectroscopic, in: J.G. Webster (Ed.). Wiley Encyclopedia of Electrical and Electronics Engineering, John Wiley and Sons, Inc., 2000.

[17] H. Mueller. Memorandum on the polarization optics of the photoelastic shutter. Technical Report 2 of the OSSRD project OEMsr-576, 1943.

[18] H. Arwin. Thin Film Optics and Polarized Light. Hans Arwin, the aureus edition, 2014.

[19] J.C. Stover. Optical Scattering: Measurement and Analysis. McGraw-Hill, Inc., 1990.

[20] R.M.A Azzam. Photopolarimetric measurement of the Mueller matrix by Fourier analysis of a single detected signal. Opt. Lett, 2:148-150, 1978.

[21] B. Pradhan and A. J. Pal. Organic heterojunction photovoltaic cells: role of functional groups in electron acceptor materials. Solar Energy Materials and Solar Cells, 81(4):469 - 476, 2004.

[22] F.L.E. Jakobsson, X. Crispin, M. Cölle, M. Büchel, D.M. de Leeuw, and M. Berggren. On the switching mechanism in Rose Bengal-based memory devices. Org. Electron, 8:559-565, 2007.

[23] S. Jönsson. Towards flexible organic electronics : photoelectron spectroscopy of surfaces and interfaces. Dissertation, Linköping University, ITN, Studies in Science and Technology, 2004.

[24] N.-K. Persson. Optical modelling of conjugated polymers - from materials to devices. Dissertation, Linköping University, IFM, Applied Physics, Biomolecular and Organic Electronics, 2005.

[25] H. Arwin, R. Magnusson, J. Landin, and K. Järrendahl. Chirality-induced polarization effects in the cuticle of scarab beetles: 100 years after Michelson. Phil. Mag., 92:1583-1599, 2012.

[26] P. Vukusic, B. Hallam, and J. Noyes. Brilliant whiteness in ultrathin beetle scales. Science, 315:348, 2007. 
[27] K. Yu, T. Fan, S. Lou, and D. Zhang. Biomimetic optical materials: Integration of nature's design for manipulation of light. Progress in Materials Science, 58:825-873, 2013.

[28] A. Boardman. Pioneers in metamaterials: John Pendry and Victor Veselago. Journal of Optics, 13(2):020401, 2011. 


\section{Papers}

The articles associated with this thesis have been removed for copyright reasons. For more details about these see:

http://urn.kb.se/resolve?urn=urn:nbn:se:liu:diva-115369 